\title{
Diagnosis and treatment of gastroesophageal reflux disease in infants and children
}

Yvan Vandenplas MD PhD

Y Vandenplas. Diagnosis and treatment of gastroesophageal reflux disease in infants and children. Can J Gastroenterol 2000;14(Suppl D):26D-34D. Gastroesophageal reflux is a frequent, nonspecific phenomenon in infants and children. The recommended approach for infants with uncomplicated regurgitation is the reassurance of the parents about the physiological nature of excessive regurgitation, and if necessary, completed with dietary recommendations for formula-fed infants. If, despite these efforts, the symptoms persist, the administration of prokinetics such as cisapride is recommended before investigations such as esophageal $\mathrm{pH}$ monitoring are begun. Cisapride is the drug of choice because it has the best efficacy and safety profile. In infants and children presenting with symptoms that suggest esophagitis, endoscopy of the upper gastrointestinal tract is recommended. If there is severe esophagitis, acid suppression with $\mathrm{H}_{2}$ receptor antagonists or proton pomp inhibitors is recommended, eventually in combination with prokinetics. In life-threatening situations, or in patients who are resistant to or dependent on acid suppressive medication, a surgical procedure such as laparoscopic Nissen should be considered.

Esophageal $\mathrm{pH}$ monitoring is recommended to document gastroesophageal reflux disease in children presenting with unusual presentations such as chronic respiratory disease. Treatment consists of prokinetics and/or acid suppressive drugs, and surgery should be considered in many of these patients.

Key Words: Acid suppression; Endoscopy; Esophagitis; Gastroesophageal reflux; $\mathrm{H}_{2}$ receptor antagonist; Regurgitation; $\mathrm{pH}$ monitoring; Prokinetic therapy; Proton pomp inhibitor

\section{Diagnostic et traitement du reflux gastro-œsophagien chez le nourrisson et l'enfant}

RÉSUMÉ : Le reflux gastro-œsophagien (RGO) est un phénomène fréquent et non spécifique chez le nourrisson et l'enfant. En présence d'une régurgitation non compliquée chez le nourrisson, on recommande de simplement rassurer les parents et, au besoin, si le nourrisson reçoit du lait maternisé, de leur faire quelques recommandations alimentaires. Si les symptômes persistent malgré ces efforts, on recommande d'administrer un agent procinétique comme le cisapride avant de procéder à des examens comme la surveillance du pH de l'œsophage. Le cisapride est le médicament à utiliser en première intention, car c'est lui qui offre le meilleur profil d'efficacité et d'innocuité. Chez le nourrisson et l'enfant qui présentent des symptômes évocateurs d'une œsophagite, on recommande l'endoscopie des voies digestives supérieures. En présence d'une œsophagite sévère, on recommande le recours à un antagoniste des récepteurs $\mathrm{H}_{2}$ ou à un inhibiteur de la pompe à protons, possiblement en association avec un agent procinétique, pour supprimer la sécrétion acide. Dans les situations où le pronostic vital est menacé ou en cas de résistance ou de dépendance aux inhibiteurs de la sécrétion acide, il faut envisager une intervention chirurgicale comme la laparoscopie selon la méthode de Nissen. La surveillance du $\mathrm{pH}$ œsophagien est recommandée pour objectiver le RGO chez les enfants qui présentent un tableau clinique inhabituel, p. ex., une maladie respiratoire chronique. Le traitement consiste alors en l'administration d'un agent procinétique ou d'inhibiteurs de la sécrétion acide; la chirurgie doit être envisagée chez un grand nombre de ces patients.

This mini-review was prepared from a presentation made at the World Congress of Gastroenterology, Vienna, Austria, September 6 to 11, 1998 Academisch Ziekenhuis Kinderen, Vrije Universiteit Brussel, Brussels, Belgium

Correspondence and reprints: Dr Y Vandenplas, Academic Children's Hospital, Free University of Brussels, Laarbeeklaan 101, 1090, Brussels,

Belgium. Telephone+00-32-2-477-57-80, fax+00-32-2-477-57-83, e-mail yvan.vandenplas@az.vub.qc.bc

Received for publication June 18, 1999. Accepted June 23, 1999 
G astroesophageal reflux (GER) is a physiological phenomenon that occurs occasionally in every human being, especially during the postprandial period. Regurgitation occurs daily in almost $70 \%$ of four-month-old infants, and about $25 \%$ of parents consider regurgitation 'a problem' $(1,2)$. It seems illogical that the normal function of the stomach would be to reflux ingested material back into the esophagus. Whether all infants presenting with regurgitation need drug treatment is a different question.

\section{DEFINITIONS}

GER is best defined as the involuntary passage of gastric contents into the esophagus. The origin of the gastric contents can vary and includes saliva, ingested foods and drinks, and gastric, pancreatic or biliary secretions. Vomiting is used as a synonym for emesis, and means that the refluxed material comes out of the mouth 'with a certain degree of strength' or 'more or less vigorously', usually involuntary and with sensation of nausea. The term 'regurgitation' is used if the reflux dribbles effortlessly into or out of the mouth, and mostly is restricted to that occurring in infancy (from birth to 12 months) (2,3). Vomiting can be regarded as the tip of the iceberg in its relation to the incidence of GER episodes.

\section{CLINICAL PRESENTATION}

Symptoms of reflux may be observed incidentally in normal individuals; however, they occur more often and are more severe in pathological situations. The usual manifestations and unusual presentations of gastroesophageal reflux disease (GERD) are listed in Table 1 (3).

Emesis and regurgitation are the most common symptoms of primary GERD but are also manifestations of many other diseases (2,3). Secondary GERD can be caused by infections such as urinary tract infection, gastroenteritis, metabolic disorders and especially food allergy $(2,4)$. Secondary reflux may be difficult to separate clinically from primary reflux. Secondary reflux is the result of a stimulation of the vomiting centre in the dorsolateral reticular formation by many efferent and afferent impulses such as visual stimuli, the olfactory epithelium, labyrinths, pharynx, gastrointestinal and urinary tracts, and testes. Secondary GER is not discussed further in this paper. Treatment of primary GERD should focus on motility and/or acid suppression, and therapeutic management of secondary GER should focus on the etiological phenomenon.

\section{PATIENT GROUPS}

The following approach is a generalization that, like all generalizations, may need to be modified for each individual patient (3). First, interest is focused on uncomplicated GER, mostly restricted to regurgitating infants. Optimal management in patients with complicated GERD (symptoms suggestive for esophagitis) is proposed. There is a continuum between normal infants with regurgitation and GER, and those with severe GER that leads to disability, discomfort or impairment of function. An approach for the management of patients with atypical presentations of GER is proposed.
TABLE 1

Symptoms of gastroesophageal reflux disease

Usual manifestations
Specific manifestations
Regurgitation
Nausea
Vomiting
Symptoms possibly related to complications of GER*
Symptoms related to anemia (iron deficiency anemia)
Hematemesis and melena
Dysphagia (as a symptom of esophagitis or due to stricture
formation)
Weight loss and/or failure to thrive
Epigastric or retrosternal pain
Noncardiac, angina-like' chest pain
Pyrosis or heartburn, pharyngeal burning
Belching, postprandial fullness
Irritable esophagus
General irritability (infants)
Unusual manifestations
GER related to chronic respiratory diseases (bronchitis, asthma,
laryngitis, pharyngitis, etc)
Sandifer Sutcliffe syndrome
Rumination
Apnea, apparent life-threatening event and sudden infant death
syndrome
Manifestations associated with congenital and/or central nervous
system abnormalities
Intracranial tumours, cerebral palsy, psychomotory retardation

*A number of these symptoms may also be caused by other mechanisms. GER Gastroesphageal reflux

\section{GROUP 1: UNCOMPLICATED REFLUX - REGURGITATION}

Regurgitation may occur in children who are normal and do not have complaints of GERD such as nutritional deficits, esophagitis, blood loss, structures, apnea or airway manifestations. There is no difference in the incidence of regurgitation between breastfed and formula-fed infants (5). However, infants with uncomplicated regurgitation frequently are perceived by their parents as having a problem, and their parents often seek medical attention. The treatment approach of the infant presenting with excessive regurgitation and for his or her parents must be well balanced, and cannot be subject to overconcern or disregard. This group of patients is restricted mostly to infants younger than six months, or at the most 12 months $(1,3,5)$. A careful evaluation of history, observation of feeding and physical examination of the infant are mandatory; however, the possibility has not been validated thoroughly because randomization is not possible (only anxious parents seek medical help). It is rather unlikely that regurgitation will result in severe GERD. Many parents who have a regurgitating infant are anxious about the pathophysiological mechanism and prognosis. Parents especially fear long term complications if the regurgitation is not stopped. Therefore, it is important to have a long discussion with the parents to learn the frequency and volume of 


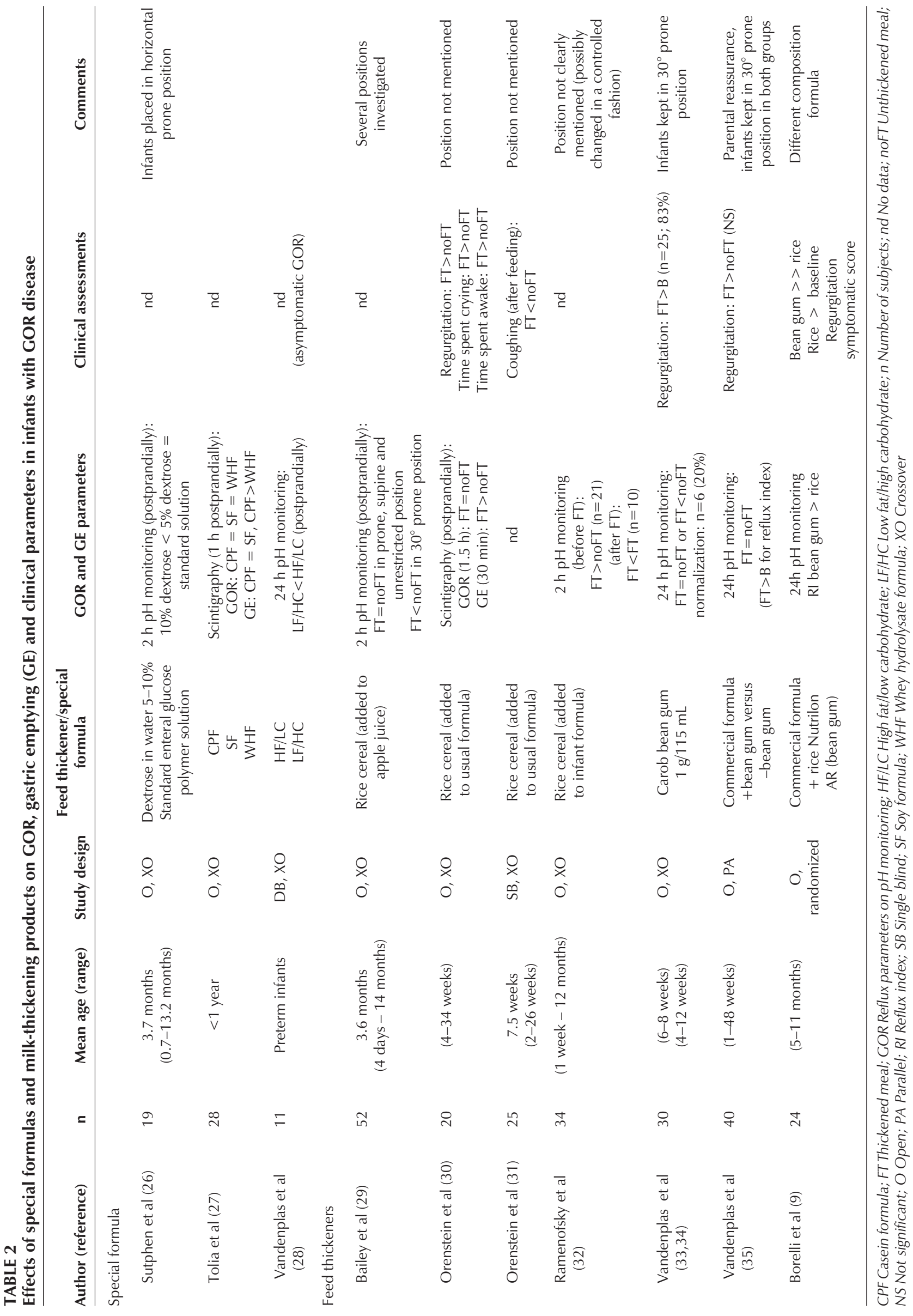


feedings, how the baby is handled during and after feedings, etc. It is also very important to explain the normal, physiological origin of regurgitation. It has been observed in several studies, that just by reassuring the parents and giving them some very practical advice on how to feed the infant is helpful $(6,7)$. The effects of parental reassurance have been suggested by many placebo controlled studies to show similar efficacies between the placebo and the tested intervention $(6,7)$. If simple reassurance fails, dietary intervention is recommended, including restriction of the volume in overfed babies, and change to a thickened 'antiregurgitation' formula (5-7). Larger food volumes and high osmolality increase the number of transient lower esophageal sphincter (LES) relaxations and decrease LES pressure to almost undetectable levels (8). Both are well known pathophysiological mechanisms that provoke GER in infants, which may also explain why feed thickeners sometimes aggravate the symptoms. Thickening the formula with starch (eg, from rice or potato) or non-nutritive thickeners (bean gum) decreases the frequency and volume of regurgitation (5-7,9)(Table 2). Some of these 'antiregurgitation' formulas are caseinpredominant (casein/whey; 80\%/20\%) to optimize the curd formation, while others contain $100 \%$ whey hydrolysate to enhance gastric emptying. However, the effect of these formulas on GER parameters, when measured with $\mathrm{pH}$ monitoring or scintigraphy, is not convincing; most studies showed that reflux parameters improved, remained unchanged or worsened in approximately one of three infants for each possibility $(6,7,10)$. In other words, 'antiregurgitation' formulas did what they claimed to do - they reduced regurgitation (5-7) but they did not influence acidic GER. Thickened formula also increased the duration of sleep $(5,6)$. Therefore, antiregurgitation formula should be considered as the first step in medical treatment and should only be available by prescription (3,5-7). Antiregurgitation formula and/or dietary intervention in general should be nutritionally safe (11). However, regurgitation may be part of the spectrum of symptoms of GERD, necessitating an effective intervention to decrease the number and intensity of the GER episodes. In this situation, an intervention that is limited to alleviating the presenting manifestation (regurgitation) will not suffice. Clinical differentiation between regurgitation and (pathological) vomiting can be difficult because there is a continuum between both conditions (5). It is not always obvious in this patient group whether the parental complaints relate to physiological regurgitation or whether they suggest GERD. In practice, feed thickeners or special formula cannot be given to breastfed infants. Therefore, if the infant is breastfed and/or has GERD, drug treatment with prokinetics should be considered before diagnostic procedures.

It seems reasonable to add medication such as prokinetics to the treatment of cases that are refractory to dietary intervention. They reduce regurgitation via their effects on the LES pressure and motility, esophageal peristalsis and gastric emptying (12). For this reason, they interact with the pathophysiological mechanisms of regurgitation in infants, which are related to immaturity of the gastroesophageal motor function (13). A link between cisapride and increased salivary secretion has been demonstrated (14). This indicates that, in combination with increased peristalsis and hence esophageal clearance, cisapride therapy may protect the esophagus via salivary components, such as bicarbonate and nonbicarbonate buffers, thus facilitating symptomatic relief and healing of the esophagus. Metoclopramide and domperidone have antiemetic properties due to their dopamine receptor blocking activity, whereas cisapride is a prokinetic agent acting through indirect release of acetylcholine in the myenteric plexus (12). Although all three agents have been shown to reduce regurgitation in infants $(6,7)$, data for cisapride are more convincing (Tables 3,4$)$. Compared with metoclopramide, cisapride may be more effective in reducing $\mathrm{pH}$-metric parameters (15), has a faster onset of action (16) and is better tolerated (16). Cisapride has also been shown to heal esophagitis (17). Domperidone has been reported to be as effective as metoclopramide (18) (and thus less effective than cisapride). Extrapyramidal reactions and increased prolactine levels are effects related to the dopamine receptorblocking activity of these drugs. In the case of cisapride, which is devoid of dopamine-blocking properties at therapeutic doses, the most common adverse effects are transient diarrhea and colic (in about 2\%) (12,19). Isolated incidents of more serious adverse reactions such as side effects on the central nervous system, extrapyramidal reactions and seizures (in epileptic patients), cholestasis (in extreme premature infants) and cardiac interactions have been reported. Cisapride is metabolized by cytochrome P450 3A4 and has the potential to prolong the QT interval (19). However, an extensive review of the literature resulted in reassuring safety consensus statements (19). Serious cardiac adverse reactions have not been reported in patients treated with a dosage within the recommended regimen $(0.8 \mathrm{mg} / \mathrm{kg} / \mathrm{day}$; $40 \mathrm{mg} /$ day maximum) and in the absence of any of the additional risk factors (Table 4). Cisapride should not be taken with systemic or oral azole antifungals, or with macrolides. Both azole antifungals and macrolides interact with cytochrome P450 3A4, resulting in elevated cisapride plasma levels. In view of its mode of action, efficacy and safety, as well as its lower or equal cost compared with that of other therapeutic agents for GER, cisapride is recommended when dietary treatment fails or in regurgitating breastfed infants, if therapy is indicated. It merits consideration that prokinetics stimulate a physiological activity (peristalsis), while acidsuppressive medication inhibits a physiological secretion.

In the nonbreastfed infant, a change to a thickened hydrolysate or amino acid formula should be considered if regurgitation is resistant to a thickened formula with normal proteins or to prokinetics, because protein allergy may present as therapy-resistant GERD.

Non-drug treatment (positional therapy, dietary advice) can help to convince parents of the physiological nature of regurgitations (3). The influence of position on the incidence and duration of GER episodes has been demonstrated in adults, children and infants, both in asymptomatic 
TABLE 3

Effects of cisapride (CIS) on GOR disease in infants

\begin{tabular}{|c|c|c|c|c|c|c|c|c|}
\hline Study & $\mathbf{n}$ & $\begin{array}{l}\text { Mean age } \\
\text { (range) }\end{array}$ & $\begin{array}{l}\text { Study } \\
\text { design }\end{array}$ & Treatment & $\begin{array}{c}\text { Dietary } \\
\text { measures }\end{array}$ & $\begin{array}{l}\text { GOR and GE } \\
\text { parameters }\end{array}$ & $\begin{array}{c}\text { Clinical } \\
\text { assessments }\end{array}$ & Comments \\
\hline \multirow[t]{2}{*}{$\begin{array}{l}\text { Brueton et al } \\
\qquad(36)\end{array}$} & 7 & nd & $\mathrm{O}$ & $\begin{array}{l}\text { CIS } 0.2 \mathrm{mg} / \mathrm{kg} \text { tid } \\
\text { (3 weeks) }\end{array}$ & nd & $\begin{array}{c}\text { 24h pH monitoring: } \\
\mathrm{CIS}=\mathrm{B} \text { except for } \\
\text { number of episodes: } \\
\text { CIS }>\mathrm{B}\end{array}$ & nd & $\begin{array}{c}\text { Neurologically impaired } \\
\text { children ( } 6 \text { cerebral } \\
\text { palsy, } 1 \text { Down } \\
\text { syndrome })\end{array}$ \\
\hline & 15 & nd & $\mathrm{O}$ & $\begin{array}{l}\text { CIS } 0.2 \mathrm{mg} / \mathrm{kg} \text { tid } \\
\text { (3 weeks) }\end{array}$ & nd & $\begin{array}{c}24 \mathrm{~h} \text { pH monitoring: } \\
\mathrm{CIS}>\mathrm{B}\end{array}$ & nd & \\
\hline $\begin{array}{l}\text { Carrasco et al } \\
\quad(37)\end{array}$ & 34 & $\begin{array}{l}\text { (4 months } \\
\text { to two years) }\end{array}$ & $\mathrm{O}$ & $\begin{array}{l}\text { CIS } 0.2 \mathrm{mg} / \mathrm{kg} \text { tid } \\
\quad(3 \text { months })\end{array}$ & nd & $\begin{array}{c}24 \text { h pH monitoring: } \\
\text { CIS }>\text { B }\end{array}$ & $\begin{array}{c}\text { Symptoms: } \mathrm{CIS}>\mathrm{B} \\
\text { Endsocopy/histology: } \\
\mathrm{CIS}>\mathrm{B}\end{array}$ & \\
\hline $\begin{array}{l}\text { Carroccio et al } \\
\quad(38)\end{array}$ & 20 & $\begin{array}{l}8 \text { months } \\
\text { (3-13 months) }\end{array}$ & $\mathrm{O}$ & $\begin{array}{l}\text { CIS } 0.33 \mathrm{mg} / \mathrm{kg} \text { tid } \\
\quad(8 \text { weeks })\end{array}$ & & $\begin{array}{c}24 \text { h pH monitoring: } \\
\text { CIS }>\text { B } \\
\text { Ultrasound (GE): CIS }>\text { B }\end{array}$ & nd & $\begin{array}{c}\text { Compared with } \mathrm{CO} \\
\text { group without } \mathrm{GOR} \text { : } \\
\mathrm{B}<<\mathrm{CO} ; \mathrm{CIS}=\mathrm{CO}\end{array}$ \\
\hline Castro et al (39) & 30 & $\begin{array}{l}\text { (3 months to } \\
5 \text { years) }\end{array}$ & $\mathrm{DB}, \mathrm{PA}$ & $\begin{array}{l}\text { CIS } 0.2 \mathrm{mg} / \mathrm{kg} \mathrm{bm} \\
\text { PLA (2-4 weeks) }\end{array}$ & nd & $\begin{array}{c}24 \text { h pH monitoring: } \\
\text { CIS > PLA }\end{array}$ & $\begin{array}{c}\text { Symptoms: } \mathrm{CIS}>\mathrm{PLA} \\
\text { Respiratory } \\
\text { symptoms: } \mathrm{CIS}>\mathrm{PLA}\end{array}$ & \\
\hline $\begin{array}{l}\text { Cucchiara et al } \\
\qquad(40)\end{array}$ & 17 & $\begin{array}{c}24.5 \text { months } \\
\text { (2.5-47 months) }\end{array}$ & $\mathrm{DB}, \mathrm{PA}$ & $\begin{array}{l}\text { CIS } 0.33 \mathrm{mg} / \mathrm{kg} \text { tid } \\
\text { PLA ( } 12 \text { weeks) }\end{array}$ & nd & $\begin{array}{c}\text { Manometry }(\mathrm{LOSP}) \text { : } \\
\mathrm{CIS}=\mathrm{PLA}=\mathrm{B} ; \\
\text { (peristalsis): } \mathrm{CIS}>\mathrm{B} \\
5 \mathrm{~h} \text { pH monitoring } \\
\text { (postprandially after } \\
\text { apple juice): } \mathrm{CIS}>\mathrm{B} \text {; } \\
\mathrm{PLA}=\mathrm{B}\end{array}$ & $\begin{array}{c}\text { Symptoms: } \mathrm{CIS}>\mathrm{PLA} \\
\text { Histology: } \mathrm{CIS}>\mathrm{B} ; \\
\text { PLA }=\mathrm{B} \\
\text { Endoscopy: } \mathrm{CIS}>\mathrm{PLA}\end{array}$ & $\begin{array}{c}\text { Infants with peptic } \\
\text { esophagitis } \\
\text { (normal basal LES } \\
\text { pressure) }\end{array}$ \\
\hline \multirow[t]{2}{*}{$\begin{array}{l}\text { Cucchiara et al } \\
\text { (41) }\end{array}$} & 14 & $\begin{array}{c}15.7 \\
\text { (2-38 months) }\end{array}$ & $\mathrm{DB}, \mathrm{PA}$ & $\begin{array}{l}\text { CIS } 0.15 \mathrm{mg} / \mathrm{kg} \\
\text { PLA (intravenous, } \\
\text { single) }\end{array}$ & $\mathrm{FT}$ & $\begin{array}{l}\text { Manometry (LOSP, } \\
\text { peristalsis): CIS > PLA }\end{array}$ & nd & $\begin{array}{l}\text { Single, intravenous } \\
\text { administration }\end{array}$ \\
\hline & 24 & & $\mathrm{O}, \mathrm{PA}$ & $\begin{array}{l}\text { CIS } 0.2 \mathrm{mg} / \mathrm{kg} \text { tid } \\
\text { CO (4-6 weeks) }\end{array}$ & FT & $\begin{array}{c}24 \text { h pH monitoring: } \\
\text { CIS >CO } \\
\text { normalization: } \mathrm{CIS}>\mathrm{CO}\end{array}$ & Symptoms: $\mathrm{CIS}>\mathrm{CO}$ & $\begin{array}{c}\text { Both groups receiving } \\
\text { postural and dietary } \\
\text { treatment }\end{array}$ \\
\hline Daoud et al (42) & 9 & $\begin{array}{c}83 \text { days } \\
\text { (6-150 days) }\end{array}$ & $\mathrm{O}$ & $\begin{array}{l}\text { CIS } 0.2 \mathrm{mg} / \mathrm{kg} \text { qid } \\
\quad(3 \text { months })\end{array}$ & nd & $\begin{array}{c}24 \mathrm{~h} \text { pH monitoring: } \\
\mathrm{CIS}>\mathrm{B} \\
\text { (both upright and } \\
\text { seated) }\end{array}$ & nd & $\begin{array}{c}\text { Infants with GOR and } \\
\text { apnea }\end{array}$ \\
\hline Daoud et al (43) & 42 & $\begin{array}{l}2.6 \text { years } \\
(12 \text { days }- \\
12 \text { years })\end{array}$ & $\mathrm{O}$ & $\begin{array}{l}\text { CIS } 0.2 \mathrm{mg} / \mathrm{kg} \text { tid } \\
\quad(3 \text { months })\end{array}$ & nd & $\begin{array}{c}24 \text { h pH monitoring: } \\
\text { CIS }>\text { B } \\
\text { (both upright and } \\
\text { seated) }\end{array}$ & $\begin{array}{c}\text { Respiratory } \\
\text { symptoms: } \mathrm{CIS}>\mathrm{B}\end{array}$ & $\begin{array}{l}\text { Infants with } \\
\text { GOR-related chronic } \\
\text { respiratory symptoms }\end{array}$ \\
\hline Evans et al (44) & 22 & $\begin{array}{l}7 \text { months } \\
\text { (2-44 months) }\end{array}$ & $\mathrm{DB}, \mathrm{PA}$ & $\begin{array}{c}\text { CIS } 0.2 \mathrm{mg} / \mathrm{kg} \\
\text { qid + GAV } \\
\text { CIM } 5 \mathrm{mg} / \mathrm{kg} \\
\text { qid + GAV (6 weeks) }\end{array}$ & nd & $\begin{array}{c}24 \mathrm{~h} \mathrm{pH} \text { monitoring: } \\
\mathrm{CIS}=\mathrm{CIM}\end{array}$ & & $\begin{array}{c}\text { Wide variation in GOR } \\
\text { variables; } 62 \% \text { improved } \\
\text { with CIS versus } 50 \% \\
\text { with CIM }\end{array}$ \\
\hline $\begin{array}{l}\text { Greally et al } \\
(45)\end{array}$ & 50 & (2-18 months) & $\mathrm{O}, \mathrm{PA}$ & $\begin{array}{c}\text { CIS } 0.2 \mathrm{mg} / \mathrm{kg} \text { qid } \\
(-\mathrm{FT}) \\
\text { GAV (half sachet/ } \\
90 \mathrm{~mL} \text { feed) } \\
+ \text { bean gum (=FT) } \\
\text { (4 weeks) }\end{array}$ & $\pm \mathrm{FT}$ & $\begin{array}{l}24 \mathrm{~h} \text { pH monitoring: } \\
\mathrm{CIS}-\mathrm{FT}=\mathrm{GAV}+\mathrm{FT}\end{array}$ & $\begin{array}{c}\text { Symptoms: } \\
\mathrm{CIS}-\mathrm{FT}=\mathrm{GAV}+\mathrm{FT}\end{array}$ & $\begin{array}{l}\text { Design misleading: CIS } \\
\text { without FT, GAV with FT }\end{array}$ \\
\hline lacono et al (46) & 25 & $\begin{array}{l}16.2 \text { months } \\
\text { (1 month - } \\
7 \text { years) }\end{array}$ & $\mathrm{O}$ & $\begin{array}{l}\text { CIS } 0.33 \mathrm{mg} / \mathrm{kg} \text { tid } \\
\quad(8 \text { weeks })\end{array}$ & nd & $\begin{array}{c}24 \text { h pH monitoring: } \\
\text { CIS }>\text { B }\end{array}$ & Symptoms: CIS>B & \\
\hline $\begin{array}{l}\text { Malfroot et al } \\
\quad(47)\end{array}$ & 38 & $\begin{array}{c}26 \text { months } \\
\text { (2 weeks }-7 \text { years) }\end{array}$ & $\mathrm{O}$ & $\begin{array}{l}\text { CIS } 0.3 \mathrm{mg} / \mathrm{kg} \mathrm{bm} \\
\quad(6 \text { months })\end{array}$ & nd & $\begin{array}{c}\text { Scintigraphy: } \mathrm{CIS}>\mathrm{B} \\
24 \mathrm{~h} \mathrm{pH} \text { monitoring: } \\
\mathrm{CIS}>\mathrm{B}\end{array}$ & $\begin{array}{c}\text { Respiratory } \\
\text { symptoms: CIS }>\text { B }\end{array}$ & $\begin{array}{c}\text { Infants with } \\
\text { GOR-related respiratory } \\
\text { disease }\end{array}$ \\
\hline $\begin{array}{l}\text { Mundo et al } \\
(48)\end{array}$ & 35 & (1-36 months) & $\mathrm{DB}, \mathrm{PA}$ & $\begin{array}{c}\text { CIS } 0.2 \mathrm{mg} / \mathrm{kg} \mathrm{bm} \\
\mathrm{MCL} 0.2 \mathrm{mg} / \mathrm{kg} \mathrm{bm} \\
\text { (10 weeks) }\end{array}$ & nd & nd & $\begin{array}{c}\text { Symptoms: } \mathrm{CIS}>\mathrm{MCL} \\
\text { Overall response: } \\
\mathrm{CIS}>\mathrm{MCL}(\mathrm{NS})\end{array}$ & $\begin{array}{l}\text { Adverse events: } \mathrm{n}=4 \\
\text { with CIS and } \mathrm{n}=9 \text { with } \\
\text { MCL (diarrhea, } \\
\text { irritability) }\end{array}$ \\
\hline Rode et al (49) & 40 & 6.5 months & $\mathrm{O}$ & $\begin{array}{l}\text { CIS } 1 \text { mg/kg/day } \\
\text { (in } 3 \text { doses) (1 day) }\end{array}$ & SD & $\begin{array}{c}28 \text { h pH monitoring: } \\
\text { CIS }>\text { B } \\
\text { (erect, supine and } \\
\text { prone) }\end{array}$ & nd & Acute study \\
\hline
\end{tabular}


TABLE 3 (continued from page 30D) Effects of cisapride (CIS) on GOR disease in infants

\begin{tabular}{|c|c|c|c|c|c|c|c|c|}
\hline Study & $\mathbf{n}$ & $\begin{array}{l}\text { Mean age } \\
\text { (range) }\end{array}$ & $\begin{array}{l}\text { Study } \\
\text { design }\end{array}$ & Treatment & $\begin{array}{c}\text { Dietary } \\
\text { measures }\end{array}$ & $\begin{array}{l}\text { GOR and GE } \\
\text { parameters }\end{array}$ & $\begin{array}{c}\text { Clinical } \\
\text { assessments }\end{array}$ & Comments \\
\hline Rode et al (14) & 18 & 6.5 months & $\mathrm{O}, \mathrm{XO}$ & $\begin{array}{c}\text { CIS } 0.33 \mathrm{mg} / \mathrm{kg} \text { tid } \\
\text { MCL } 0.2 \mathrm{mg} / \mathrm{kg} \text { tid } \\
\text { (1 day) }\end{array}$ & SD & $\begin{array}{c}28 \text { h pH monitoring: } \\
\mathrm{CIS}>\mathrm{MCL} \\
\text { long lasting } \mathrm{COR}, \\
\text { clearance: } \mathrm{CIS}>\mathrm{MCL} \\
\% \text { time, number of } \\
\text { episodes: } \mathrm{CIS}=\mathrm{MCLB}\end{array}$ & nd & $\begin{array}{l}\text { Acute study } \mathrm{CIS}>\mathrm{MCL} \\
\text { in all positions (erect, } \\
\text { supine and prone) }\end{array}$ \\
\hline Rode et al (50) & 30 & 10 months & $\mathrm{O}$ & $\begin{array}{l}\text { CIS } 1 \mathrm{mg} / \mathrm{kg} / \text { day } \\
\text { (in } 3 \text { doses) } \\
\text { (3 weeks) }\end{array}$ & nd & $\begin{array}{l}28 \text { h pH monitoring: } \\
\mathrm{CIS}>\mathrm{B} \text { (erect, supine, } \\
\text { prone) }\end{array}$ & Symptoms: CIS>B & \\
\hline Saye et al (51) & 14 & $\begin{array}{c}29 \text { months } \\
\text { (4 months }-11 \text { years) }\end{array}$ & $\mathrm{DB}, \mathrm{PA}$ & $\begin{array}{c}\text { CIS } 0.3+ \\
0.15 \mathrm{mg} / \mathrm{kg} / 4 \mathrm{~h} \\
\text { PLA (1 day) }\end{array}$ & nd & $\begin{array}{c}16 \text { h pH monitoring: } \\
\mathrm{CIS}>\mathrm{PLA} \\
\text { (except number of } \\
\text { episodes: } \mathrm{CIS}=\mathrm{PLA} \text { ) }\end{array}$ & nd & $\begin{array}{l}\text { Older children with } \\
\text { GOR-related chronic } \\
\text { respiratory symptoms }\end{array}$ \\
\hline Saye et al (52) & 19 & $\begin{array}{c}7 \text { years } \\
\text { (3 months }-10 \text { years) }\end{array}$ & $\mathrm{O}$ & $\begin{array}{l}\text { CIS } 0.3 \mathrm{mg} / \mathrm{kg} \text { tid } \\
\text { (4 weeks) }\end{array}$ & nd & $\begin{array}{c}24 \text { h pH monitoring: } \\
\text { CIS }>\text { B } \\
\text { (except number of } \\
\text { episodes: } \mathrm{CIS}=\mathrm{BA} \text { ) }\end{array}$ & nd & $\begin{array}{l}\text { Older children with } \\
\text { GOR-related chronic } \\
\text { respiratory symptoms }\end{array}$ \\
\hline $\begin{array}{l}\text { Vandenplas et } \\
\text { al (53) }\end{array}$ & 22 & (4-22 weeks) & $\mathrm{O}$ & $\begin{array}{l}\text { CIS } 0.2 \mathrm{mg} / \mathrm{kg} \text { qid } \\
\text { (13-16 days) }\end{array}$ & nd & $\begin{array}{c}24 \text { h pH monitoring: } \\
\text { CIS }>\text { B (asleep, awake, } \\
\text { postcibal, fasted) }\end{array}$ & $\begin{array}{l}\text { Belching, cough, } \\
\text { nocturnal wheezing, } \\
\text { irritability: CIS }>\text { B } \\
\text { Sleep dysfunction: } \\
\text { CIS }>\text { B }\end{array}$ & $\begin{array}{l}\text { Infants with irregular } \\
\text { sleep pattern } \\
\text { Simultaneous positional } \\
\text { treatment }\left(30^{\circ} \text { prone }\right)\end{array}$ \\
\hline $\begin{array}{l}\text { Vandenplas et } \\
\text { al (54) }\end{array}$ & 29 & (2-4 months) & $\mathrm{DB}, \mathrm{PA}$ & $\begin{array}{l}\text { CIS } 0.2 \mathrm{mg} / \mathrm{kg} \text { qid } \\
\text { PLA (13-16 days) }\end{array}$ & $\mathrm{FT}$ & $\begin{array}{c}24 \text { h pH monitoring: } \\
\text { CIS > B } \\
\text { Long-lasting episodes: } \\
\text { CIS }>\text { PLA = BA }\end{array}$ & $\begin{array}{c}\text { Symptoms: CIS > PLA } \\
\text { (NS) }\end{array}$ & $\begin{array}{c}\text { Both groups placed on } \\
\text { positional and dietary } \\
\text { treatment }\end{array}$ \\
\hline \multirow[t]{3}{*}{$\begin{array}{l}\text { Van Eygen et al } \\
\text { (55) }\end{array}$} & 69 & (5-12 months) & $\mathrm{O}$ & $\begin{array}{c}\text { CIS } 0.15-0.3 \mathrm{mg} \text { tid } \\
(4 \text { weeks })\end{array}$ & $\mathrm{CF}^{*}$ & nd & $\begin{array}{l}\text { Global response: } \\
\qquad \mathrm{CIS}>\mathrm{B}\end{array}$ & \\
\hline & 23 & & $\mathrm{DB}, \mathrm{PA}$ & $\begin{array}{l}\text { CIS } 0.15 \text { mg tid } \\
\text { PLA (2-4 weeks) }\end{array}$ & $\mathrm{CF}^{*}$ & nd & $\begin{array}{c}\text { Global response: } \\
\text { CIS > PLA } \\
\text { Symptoms: CIS > PLA }\end{array}$ & \\
\hline & 45 & & $\mathrm{DB}, \mathrm{PA}$ & $\begin{array}{l}\text { CIS } 0.2-0.3 \text { mg tid } \\
\text { PLA (2-4 weeks) }\end{array}$ & $\mathrm{CF}^{*}$ & nd & $\begin{array}{c}\text { Global response: } \\
\text { CIS > PLA } \\
\text { Symptoms: } \\
\text { at } 1 \text { week: } \\
\text { CIS }(0.2 \mathrm{mg})>\text { PLA } \\
\text { at } 2 \text { weeks: } \\
\text { CIS }(0.1 \mathrm{mg})>\text { PLA }\end{array}$ & \\
\hline Scott et al (56) & 45 & (6 weeks - 2 years) & $\mathrm{DB}, \mathrm{PA}$ & $\begin{array}{l}\text { CIS } 0.2 \text { mg tid } \\
\text { PLA ( } 6 \text { weeks })\end{array}$ & nd & $\begin{array}{c}24 \mathrm{~h} \text { pH monitoring } \\
\text { CIS PLA duration reflux } \\
\text { upright,supine } \\
\text { CIS=PLA RI, n epi, } \\
\text { PLES, }\end{array}$ & $\begin{array}{l}\mathrm{CIS}=\mathrm{PLA} \\
\text { regurgitation } \\
\text { frequency; global } \\
\text { evaluation score }\end{array}$ & No side effects \\
\hline Cohen et al (57) & 95 & $>36$ months & $\mathrm{DB}, \mathrm{PA}$ & $\begin{array}{l}\text { CIS } 0.2 \text { mg tid } \\
\text { PLA ( } 2 \text { weeks) }\end{array}$ & nd & $\begin{array}{l}\text { 24h pH monitoring } \\
\text { CIS PLA RI, } \\
\mathrm{n}^{\circ}>5 \text { min, duration } \\
\text { longest episode }\end{array}$ & $\begin{array}{l}\qquad \mathrm{CIS}=\mathrm{PLA} \\
\text { crying, vomiting, } \\
\text { gagging; parental } \\
\text { global evaluation }\end{array}$ & $\begin{array}{l}\text { No stepwise } \\
\text { treatment }\end{array}$ \\
\hline
\end{tabular}

*Prior therapeutic measures continued (positional and/or dietary). Symptoms: if not specified, clinical assessment including regurgitation and/or vomiting. AA Antacid; afm After meals; B Baseline; bm Before meals/each feeding; CF Customary formula; CIM Cimetidine; CO Controls; DB Double- blind; dex Dextrose; DM Dietary measures; DO Domperidone; epi Epinephrine; FT Feed thickener; GAV Gaviscon; GE Gastric emptying; glu Glucose; GOR Reflux parameters on pH monitoring; LES Lower esophageal sphincter; LOSP Lower esophageal sphincter pressure; MCL Metoclopramide; $n$ Number of subjects; $n^{\circ}$ Number; nd No data; NS Not significant; O Open; PA Parallel; PLA Placebo; PLES Pressure lower esophageal sphincter; PN Parenteral nutrition; RI Reflux index; SD Standard diet; SF Solid food started if not yet done so; XO Crossover with washout period

healthy controls and symptomatic individuals. The $30^{\circ}$ prone reversed Trendelenburg position generally is recommended and accepted as an essential element of treatment $(3,6,7)$. However, positional treatment in practice is very difficult to apply correctly in infants and rather upsetting for the babies, because they must be tied up in their beds or cot to prevent them from sliding down under the blankets in or- der to achieve and maintain an angle of $30^{\circ}$. There is ample evidence that the prone sleeping position is a risk factor in sudden infant death, independent of overheating, smoking or way of feeding (6). Positional treatment remains, in view of its efficacy, a valid adjuvant treatment in patients not responding to other therapeutic approaches or beyond the age of sudden infant death syndrome (6). 
TABLE 4

\section{Contraindications and risk factors for the use of cisapride}

Contraindications to cisapride administration in pediatric patients

Use of medication also known to prolong the QT interval or potent CYP3A4 inhibitors, such as astemizole, fluconazole, itraconazole, ketoconazole, miconazole, eythromycin, clarithromycin, troleandomycin, nefazodone, indinavir, ritonavir, josamycin, diphemanil, terfaridine

Use of the above medications by a breastfeeding mother because secretion in mother's milk of most of these drugs is unknown

Known hypersensitivity to cisapride

Known congenital long QT syndrome or known idiopathic QT prolongation

Precautions* for cisapride administration in pediatric patients

Prematurity (a starting dose of $0.1 \mathrm{mg} / \mathrm{kg}$, 4 times daily may be used, although $0.2 \mathrm{mg} / \mathrm{kg}$ is also the normal dose for premature infants)

Hepatic or renal failure (particularly when on chronic dialysis). In these cases, it is recommended to start with $50 \%$ of the recommended dose

Uncorrected electrolyte disturbances (hypokalemia, hypomagnesemia, hypocalcemia), as may occur in premature infants, in patients with severe diarrhea, in treatment with potassium-wasting diuretics such as furosemide or acetazolamide

History of significant cardiac disease including serious ventricular arrhythmia, second- or third-degree antrioventricular block, congestive heart failure or ischaemic heart disease, QT prolongation associated with diabetes mellitus

History of sudden infant death in a sibling, and/or history of a 'serious' apparent life-threatening event in the infant or a sibling

Intracranial abnormalities, such as encephalitis or hemorrhage Grape fruit juice

*An electrocardiogram should be performed once before cisapride administration and two to three times after administration

\section{GROUP 2: OVERT GERD}

Patients with overt GERD either did not respond to previous approaches such as parental reassurance, dietary treatment and prokinetics, or presented with symptoms suggesting esophagitis (hematemesis, retrosternal and epigastric pain, etc) (Table 1 ). Therefore, an underlying anatomical malformation should be excluded, and endoscopy is the investigation of choice $(3,20)$. Upper gastrointestinal endoscopy in infants and children should only be performed by experienced and qualified physicians, and should always be done as a duodenogastroesophagoscopy (20). If the question being asked is restricted to underlying anatomical malformations, upper gastrointestinal series may be considered (20). If symptoms and/or the esophagitis do not improve despite adequate medical treatment and controlled compliance, upper gastrointestinal series should be performed to exclude anatomical problems such as gastric volvulus, intestinal malrotation or annular pancreas.

Antacids have been reported to be effective in the treatment of GER (6), although experience with their use in in- fants is limited. Their capacity to buffer gastric acid is strongly influenced by the time of administration (21) and requires multiple doses. Gaviscon (SmithKline Beecham, USA), a combination of antacid and sodium salt of alginic acid, is as effective as antacids and appears to be relatively safe because only a limited number of side effects have been reported. Occasional formation of large bezoar-like masses of agglutinated intragastric material has been reported with the use of Gaviscon, and it can increase the sodium content of the feeds to an undesirable degree, especially in preterm infants ( $1 \mathrm{~g}$ Gaviscon powder contains $46 \mathrm{mg}$ sodium, and the suspension contains twice this amount of sodium) (6).

$\mathrm{H}_{2}$ receptor antagonists, of which ranitidine is the most used, are effective in healing reflux esophagitis in infants and children (6). Many new drugs, such as misoprostil, sucralfate and omeprazole, have been developed. Of these, the proton pump inhibitors (PPIs) have been studied most effectively, although experience in infants and children is limited $(22,23)$. PPIs are effective in suppressing the acidity in patients with gastric stress ulcers and also in neurologically impaired children. Even in patients with circular esophageal ulcerations, recent experience suggests a trial of PPIs before surgery (22). Omeprazole has been shown to be effective in cases of patients with severe esophagitis refractory to $\mathrm{H}_{2}$ blockers (22). Sucralfate was shown to be as effective as cimetidine for esophagitis in children (24).

Immediate or early surgery is rarely indicated in life-threatening conditions where medical management is of no benefit. Surgery can be life-saving in severely affected patients (notably neurologically impaired children with recurrent and life-threatening aspiration). Before surgery, a full diagnostic workup including upper gastrointestinal series, endoscopy, $\mathrm{pH}$ monitoring, and manometry and gastric emptying studies is recommended.

\section{GROUP 3: PATIENTS WITH UNUSUAL PRESENTATIONS OF GER}

The most obvious difference between this patient group and groups 1 and 2 is that group 3 does not present with emesis and regurgitation (Table 1). Because these patients do not vomit, GERD is 'occult'. Before considering GER as a cause of the symptoms, classic causes of the manifestations such as allergy in a wheezing patient and tuberculosis in a patient with chronic cough must be excluded.

If GERD is suspected, $\mathrm{pH}$ monitoring of long duration (18 to $24 \mathrm{~h}$ ) is the investigation of choice. In this group of patients, $\mathrm{pH}$ monitoring may need to be performed simultaneously with other investigations in order to relate $\mathrm{pH}$ changes to events (for example, polysomnography in infants presenting with an apparent life-threatening event). In patients suspected of pulmonary aspiration, a scintigraphy might prove the association (although a negative scintigraphy does not exclude reflux-related aspiration, and the therapeutic approach is identical).

If $\mathrm{pH}$ monitoring parameters are abnormal or if events are clearly related to $\mathrm{pH}$ changes, prokinetics, eventually in combination with $\mathrm{H}_{2}$ receptor antagonists or PPIs, are indi- 
cated $(20,22)$. In this group, repeated $\mathrm{pH}$ monitoring under treatment conditions in combination with a clinical followup is mandatory. Depending on the unusual presentation, treatment can be stopped after six to 12 months because a possible mechanism for GER in association with unusual manifestations may be self-perpetuating GER (25). Once reflux occurs, acid gastric contents, containing pepsin and sometimes bile, come into contact with the esophageal mucosa, which increases the esophageal permeability to acid and makes the esophageal mucosa much more susceptible to inflammatory changes. Esophageal inflammation, even restricted to the lower esophagus, impairs LES pressure and function, and favours GER (25).

\section{SEVERELY NEUROLOGICALLY IMPAIRED CHILDREN}

The vast majority of neurologically impaired children suffer from severe GERD. Most of these children are under specialized follow-up, and only brief recommendations are given here. The pathophysiological mechanism of GERD in these children is particularly multifarious: the neurological disease itself may cause delayed esophageal clearance and gastric emptying; most of these children are bedridden (gravity improves esophageal clearance); and many children are constipated (which increases abdominal pressure and favours GER).

\section{REFERENCES}

1. Nelson SP, Chen EH, Syniar GM, Christoffel KK. Prevalence of symptoms of gastroesophageal reflux in infancy. Pediatric Practice Research Group. Arch Pediatr Adolesc Med 1997;151:569-72.

2. Orenstein S. Gastroesophageal reflux. In: Hyman PE, ed. Pediatric Gastrointestinal Motility Disorders. New York: Academy Professional Information Services, 1994:55-88

3. Vandenplas Y, Ashkenazi A, Belli D, et al. A proposition for the diagnosis and treatment of gastro-oesophageal reflux disease in children: a report from a working group on gastro-oesophageal reflux disease. Working Group of the European Society of Pediatric Gastroenterology \& Nutrition (ESIGAN). Eur J Pediatr 1993;152:704-11.

4. Carvataio F, Iacono G, Montalto G, Soresi M, Tumminello M, Carroccio A. Clinical and $\mathrm{pH}$-metric characteristics of gastro-oesophageal reflux secondary to cows' milk protein allergy. Arch Dis Child 1996;75:51-6.

5. Vandenplas Y, Lifshitz JZ, Orenstein S, et al. Nutritional management of regurgitation in infants. J Am Coll Nutr 1998;17:308-16.

6. Vandenplas Y, Belli D, Benhamou P, et al. A critical appraisal of current management practices for infant regurgitation recommendations of a working party. Eur J Pediatr 1997;156:343-57.

7. Vandenplas Y, Belli D, Cadranel S, et al. Dietary treatment for regurgitation - recommendations from a working party. Acta Paediatr 1998;87:462-8

8. Cucchiara S, De Vizia B, Minella R, et al. Intragastric volume and osmolality affect mechanisms of gastrooesophageal reflux in children with GOR disease. J Pediatr Gastroenterol Nutr 1995;20:468. (Abst)

9. Borelli O, Salvia G, Campanozzi A, et al. Use of a new thickened formula for treatment of symptomatic gastrooesophageal reflux in infants. Ital J Gastroenterol Hepatol 1997;29:237-42.

10. Vandenplas Y. Clinical use of cisapride and its risk-benefit in paediatric patients. Eur J Gastroenterol Hepatol 1998;10:871-81.

11. Levtchenko E, Hauser B, Vandenplas Y. Nutritional value of an "anti-regurgitation" formula. Acta Gastroenterol Belg 1998;61:285-7.

12. Verlinden M, Welburn P. The use of prokinetic agents in the treatment of gastro-intestinal motility disorders in childhood. In: Milla PJ, ed. Disorders of gastro-intestinal motility in childhood. Chichester: John Wiley \& Sons Ltd, 1988:125-40.

\section{CONCLUSIONS}

The diagnostic approach of GERD in infants and children principally depends on its presenting features. Infants with typical symptoms of uncomplicated GER (the majority of regurgitating babies) should be treated without prior investigations. Endoscopy, in specialized centres, is recommended if esophagitis is suspected. Long term esophageal $\mathrm{pH}$ montoring is the investigation of choice and occupies a central position in the diagnostic approach for the patient suspected of unusual or atypical presentations of GERD, such as 'occult' GERD. Nondrug treatment (the importance of parental reassurance cannot be stressed enough) and dietary treatment are effective and safe approaches in infant regurgitation therapy but do not treat GERD. If the symptoms are refractory to this approach, or in reflux disease, cisapride is the drug of choice. PPIs or $\mathrm{H}_{2}$ receptor antagonists, in combination with prokinetics, are recommended in ulcerative esophagitis. There is no excuse to persist with an ineffective management of a disease that might result in stunting, chronic illness, persistent pain, esophageal scarring or even death. Management of GERD in infants and children, therefore, should be thoroughly considered, avoiding overinvestigations and overtreatment of a self-limiting condition, but also avoiding underestimation of potential severe disease, accompanied by serious morbidity.

13. Boix-Ochoa J. The physiologic approach to the management of gastric esophageal reflux. J Pediatr Surg 1986;21:1032-9.

14. Heading RC, Baldi F, Holloway RH, et al. Prokinetics in the treatment of gastro-oesophageal reflux disease. Eur J Gastroenterol Hepatol 1998;10:87-93.

15. Rode H, Stunden RJ, Millar AJW, Cywes S. Esophageal pH assessment of gastroesophageal reflux in 18 patients and the effect of two prokinetic agents: cisapride and metoclopramide. J Pediatr Surg 1987;22:931-4.

16. Mundo F, Feregrino H, Fernandez J, Teramoto O, Abord P, et al. Clinical evaluation of gastroesophageal reflux in children: double-blind study of cisapride vs metoclopramide. Am J Gastroenterol 1990;85:A29.

17. Cucchiara S, Staiano A, Capozzi C, Di Lorenzo C, Boccieri A, Auricchio S. Cisapride for gastro-oesophageal reflux and peptic oesophagitis. Arch Dis Child 1987;62:454-7.

18. De Loore I, Van Ravensteyn H, Ameryckx L. Domperidone drops in the symptomatic treatment of chronic paediatric vomiting and regurgitation. A comparison with metoclopramide. Postgrad Med J 1979;55(Suppl 1):40-2.

19. Vandenplas Y, Belli DC, Benatar A, et al. The role of cisapride in the treatment of paediatric gastroesophageal reflux. The European Society of Paediatric Gastroenterology, Hepatology \& Nutrition. J Pediatr Gastroenterol Nutr 1999;28:518-28.

20. Vandenplas Y. Reflux esophagitis in infants and children. J Pediatr Gastroenterol Nutr 1994;18:413-22.

21. Sutphen JL, Dillard VL, Pipan ME. Antacid and formula effects on gastric acidity in infants with gastroesophageal reflux. Pediatrics 1986;78:55-7.

22. Israel DM, Hassall E. Omeprazole and other proton pump inhibitors : pharmacology, efficacy and safety, with special reference to use in children. J Pediatr Gatsroenterol Nutr 1998;27:568-79.

23. Cohen RC, O'Loughlin EV, Davidson GP, Moore DJ, Lawrence DM. Cisapride in the control of symptoms in infants with gastroesophageal reflux: a randomized, double-blind, placebo-controlled trial. J Pediatr 1999; 134:287-92.

24. Arguelles-Martin F, Gonzalez-Fernandez F, Gentles MG, Navarro-Merino M. Sucralfate in the treatment of reflux esophagitis 
in children. Preliminary results. Scand J Gastroenterol Suppl 1989;156:43-7.

25. Vandenplas Y. Physiopathological mechanisms of gastro-oesophageal reflux: is motility the clue? Rev Med Brux 1994;15:7-9.

26. Sutphen JL, Dillard VL. Dietary caloric density and osmolarity influence gastroesophageal reflux in infants. Gastroenterology 1989;97:601-4.

27. Tolia V, Lin S, Kuhns LR. Gastric emptying using three different formulas in infants with gastroesophageal reflux. J Pediatr Gastroenterol Nutr 1992;15:297-301.

28. Vandenplas Y, Sacre L, Loeb H. Effects of formula feeding on gastric acidity time and oesophageal $\mathrm{pH}$ monitoring data. Eur J Pediatr 1988;148:152-4.

29. Bailey DJ, Andres JM, Danek GD, Pineiro-Carrero VM. Lack of efficacy of thickened feeding as treatment for gastroesophageal reflux. J Pediatr 1987;110:187-9.

30. Orenstein SR, Magill HL, Brooks P. Thickening of infant feedings for therapy of gastroesophageal reflux. J Pediatr 1987;110:181-6.

31. Orenstein SR, Shalaby TM, Putman PE. Thickened feedings as a cause of increased coughing when used as therapy for gastroesophageal reflux in infants. J Pediatr 1992;121:913-5.

32. Ramenofsky ML, Leape LL. Continuous upper esophageal $\mathrm{pH}$ monitoring in infants and chidlren with gastroesophageal reflux, pneumonia and apneic spells. J Pediatr Surg 1981;16:374-8.

33. Vandenplas Y, Sacre-Smits L. Gastro-oesophageal reflux in infants: evaluation of treatment by $\mathrm{pH}$ monitoring. Eur J Pediatr 1987;146:504-7.

34. Vandenplas Y, Sacre L. Milk-thickening agents as a treatment for gastroesophageal reflux. Clin Pediatr (Phila) 1987;26:66-8.

35. Vandenplas Y, Hachimi-Idrissi S, Casteels A, Mahler T, Loeb H. A clinical trial with an "anti-regurgitation" formula. Eur J Pediatr 1994;153:419-23.

36. Brueton MJ, Clarke GS, Sandhu BK. The effects of cisapride on gastro-oesophageal reflux in children with and without neurological disorders. Dev Med Child Neurol 1990;32:629-32.

37. Carrasco S, lama R, Prieto G, Polanco I. Treatment of gastroesophageal reflux and peptic oesophagitis with cisapride. In: Heading RC, Wood JD, eds. Gatsrointestinal Dysmotility: Focus on Cisapride. New York: Raven press, 1992:326-7.

38. Carroccio A, Iacono G, Li Voti G, et al. Gastric emptying in infants with gastroesophageal reflux. Ultrasound evaluation before and after cisapride administration. Scand J Gastroenterol 1992;27:799-804.

39. Castro HE, Ferrero GB, Cortina LS, Salces C, Lima M. Effectividad del cisapride en el trtamiento del reflujo gastroesofagico (RGE) in nonos. Valoracion de un estudio a doble ciego. An Espegnol Pediatr 1994;40:5-8.

40. Cucchiara S, Staiano A, Capozzi C, Di Lorenzo C, Boccieri A, Auricchio S. Cisapride for gastro-oeosphageal reflux and peptic oesophagitis. Arch Dis Child 1987;62:454-7.

41. Cucchiara S, Staiano A, Boccieri A, et al. Effects of cisapride on parameters of oesophageal motility and on the prolonged intraoesophageal $\mathrm{pH}$ test in infants with gastro-oesophageal reflux disease. Gut 1990;31:21-5.
42. Daoud G, Gonzalez L, Medina M, et al. Efficacy of cisapride in infants with apnea and gastroesophageal reflux evaluated by prolonged intraesophageal $\mathrm{pH}$ monitoring. 2nd UEGW, Barcelona, Spain. July 19-23, 1993:A107.

43. Daoud G, Stanzione C, Abraham A, et al. Response to cisapride in children with respiratory symptoms and gastroesophageal reflux evaluated by prolonged intraesophageal $\mathrm{pH}$ monitoring. 2nd UEGW, Barcelon, Spain, July 19-23, 1993:A108.

44. Evans DF, Ledingham SJ, Kapila L. The effect of medical therapy on gastro-oesophageal reflux disease in children. World Congresses of Gastroenterology, Sydney, Australia, August 26-31, 1990:APP53.

45. Greally P, Hampton FJ, MacFadyen UM, Simpson H. Gaviscon and Carobel with cisapride in gastro-oesophageal reflux. Arch Dis Child 1992;67:618-21.

46. Iacono G, Carrocio A, Montalto G, et al. [Evaluation of the effectiveness of cisapride in the treatment of gastroesophageal reflux]. Minerva Pediatr 1992;44:613-6.

47. Malfroot A, Vandenplas Y, Verlinden M, Piepsz A, Dab I. Gastroesophageal reflux and unexplained chronic repsiratory disease in infants and children. Pediatr Pulmonol 1987;3:208-13.

48. Mundo F, Feregrino H, Fernandez J. Clinical evaluation of gastroesophageal reflux in children: double-blind study of cisapride vs metoclopramide. Am J Gastroenterol 1990;85:A29.

49. Rode H, Stunden RJ, Millar AJ, Cywes S. Esophageal pH assessment of gastroesophageal reflux in 18 patients and the effect of two prokinetic agents: cisapride and metoclopramide. Pediatr Surg 1987;22:931-4.

50. Rode H, Millar AJW, Melis J, Cewis S. Pharmacological control of gastro-oesophageal reflux with cisapride in infants: long-term evaluation. In : Heading RC, Wood JD, eds. Gastrointestinal Dysmotility: Focus on Cisapride. New York: Raven Press, 1992:325.

51. Saye Z, Forget PP. Effect of cisapride on esophageal $\mathrm{pH}$ monitoring in children with reflux-associated bronchopulmonary disease. J Pediatr Gastroenterol Nutr 1989;8:327-32.

52. Saye Z, Forget PP, Geubelle F. Effect of cisapride on gastroesophageal reflux in children with chronic bronchopulmonary disease: a double-blind cross-over $\mathrm{pH}$-monitoring study. Pediatr Pulmonol 1987;3:8-12

53. Vandenplas Y, Deneyer M, Verlinden M, Aerts T, Sacre L. Gastroesophageal reflux incidence and respiratory dysfunction during sleep in infants: treatment with cisapride. J Pediatr Gastroenterol Nutr 1989;8:31-6.

54. Vandenplas Y, de Roy C, Sacre L. Cisapride decreases prolonged episodes of reflux in infants. J Pediatr Gastroenterol Nutr 1991;12:44-7.

55. Van Eygen M, Van Ravensteyn H. Effect of cisapride on excessive regurgitation in infants. Clin Ther 1989;11:669-77.

56. Scott RB, Ferreira C, Smith L, et al. Cisapride in pediatric gastroesophageal reflux. J Pediatr Gastroenterol Nutr 1997;25:499-506.

57. Walters JK, Zimmermann AE, Souney PF, Katona BG. The use of omeprazole in the pediatric population. Ann Pharmacother $1998 ; 32: 478-81$. 


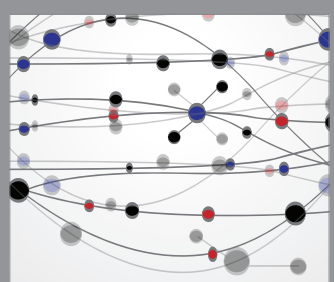

The Scientific World Journal
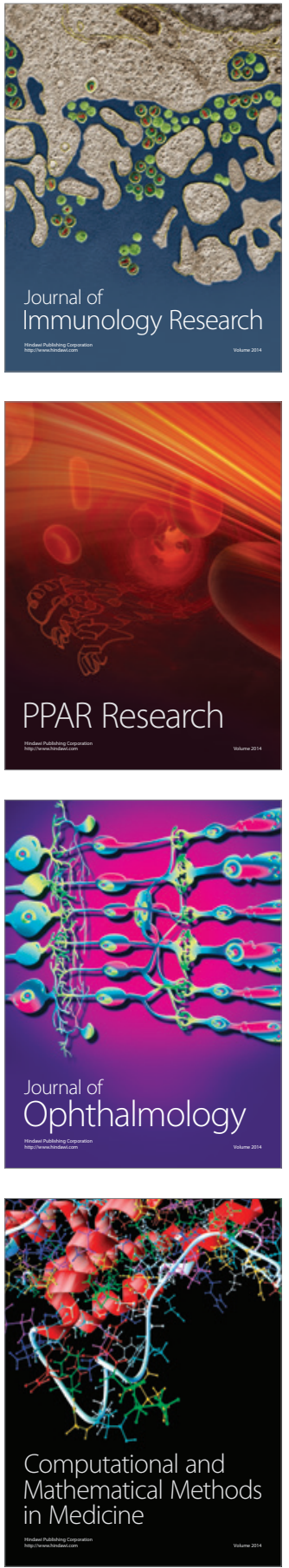

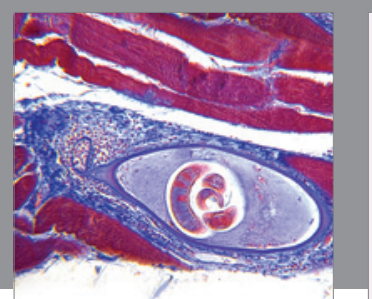

Gastroenterology Research and Practice

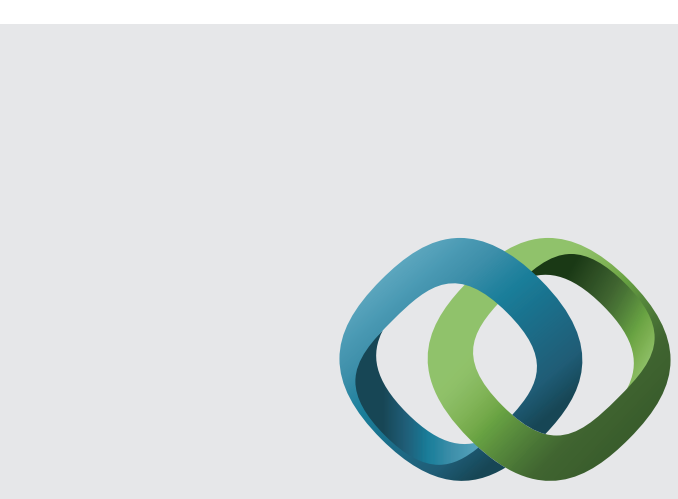

\section{Hindawi}

Submit your manuscripts at

http://www.hindawi.com
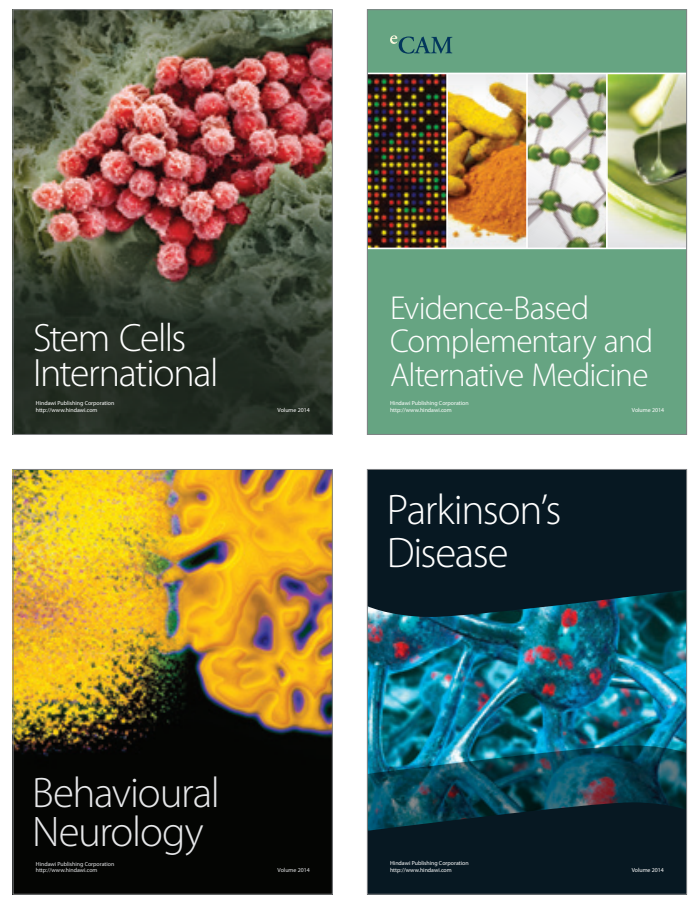
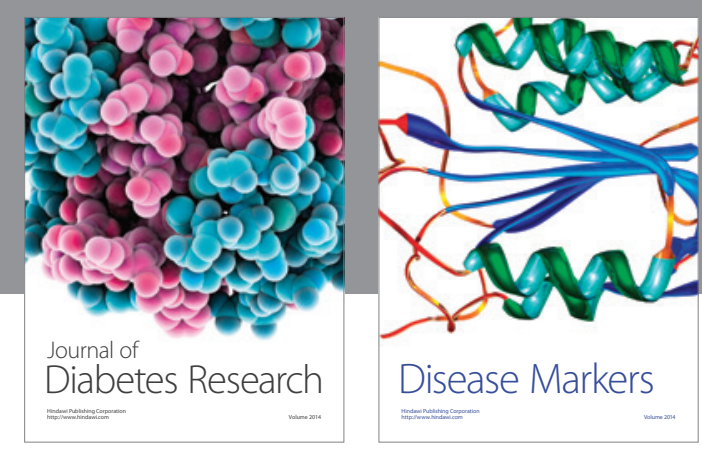

Disease Markers
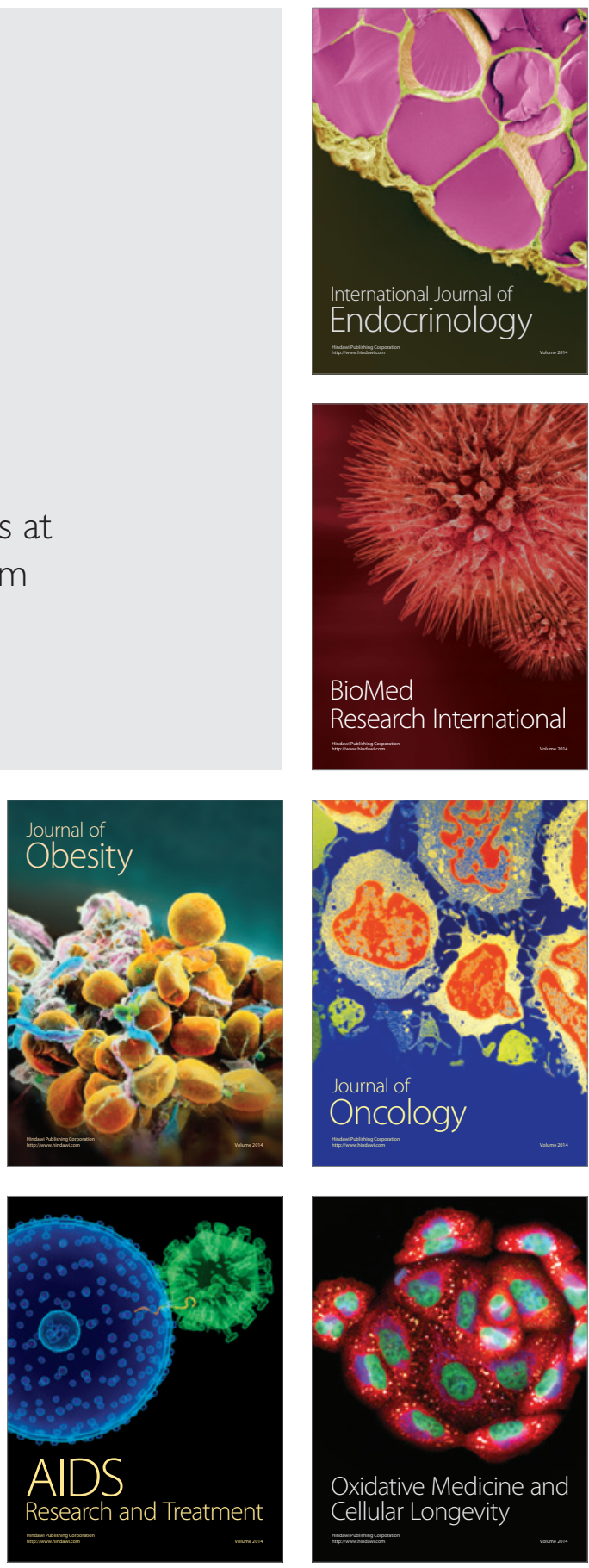